\title{
Iterative Data Squashing for Boosting Based on a Distribution-Sensitive Distance
}

\author{
Yuta Choki and Einoshin Suzuki \\ Division of Electrical and Computer Engineering \\ Yokohama National University, Japan \\ $\{$ choki, suzuki\}@slab.dnj .ynu.ac.jp
}

\begin{abstract}
This paper proposes, for boosting, a novel method which prevents deterioration of accuracy inherent to data squashing methods. Boosting, which constructs a highly accurate classification model by combining multiple classification models, requires long computational time. Data squashing, which speeds-up a learning method by abstracting the training data set to a smaller data set, typically lowers accuracy. Our SB (Squashing-Boosting) loop, based on a distribution-sensitive distance, alternates data squashing and boosting, and iteratively refines an SF (Squashed-Feature) tree, which provides an appropriately squashed data set. Experimental evaluation with artificial data sets and the KDD Cup 1999 data set clearly shows superiority of our method compared with conventional methods. We have also empirically evaluated our distance measure as well as our SF tree, and found them superior to alternatives.
\end{abstract}

\section{Introduction}

Boosting represents a learning method which constructs a highly accurate classification model by combining multiple classification models, each of which is called a weak hypothesis [4]. It is possible to reduce computational time of boosting by using data squashing [3], which decreases the number of examples in the data set, typically at the sacrifice of accuracy. In order to circumvent this problem, we propose a method of utilizing the probability distribution over training examples provided by boosting methods, here AdaBoost.M2 [4], to data squashing. Data squashing and boosting episodes are alternated in order to employ the examples' weights determined by AdaBoost.M2 to determine thresholds used by the data squashing algorithm. Moreover, we consider distribution of examples in the process by using our projected SVD distance as the distance measure for data squashing. Effects of the iterative data squashing and the distance measure are empirically evaluated through experiments with artificial and real-world data sets.

This paper is structured as follows. In section 2, we review boosting especially AdaBoost.M2 [4], which we employ throughout this paper. Section 3 explains previous research for fast learning based on data squashing. In section 4, we propose our SB (Squashing-Boosting) loop, and evaluate it through experiments in section 5. Section 6 describes concluding remarks. 


\section{Boosting}

The goal of boosting is to construct a "strong learner" which demonstrates high accuracy by combining a sequence of "weak learners" each of which has accuracy slightly higher than random learning. AdaBoost.M2 deals with a classification problem with no less than 3 classes, and constructs each weak hypothesis by transforming the original classification problem to a binary classification problem in terms of an original class. AdaBoost.M2 utilizes, maintains, and modifies an example weight for each example and a model weight for each weak hypothesis. An example weight represents the degree of importance for the example in constructing a weak hypothesis, and is initialized uniformly before learning the first weak hypothesis. An example weight is increased when the obtained weak hypothesis misclassifies the example, and vice versa. A model weight represents the degree of correctness of the corresponding weak hypothesis. We describe a brief outline of AdaBoost.M2 below.

A training data set $\left(\mathbf{x}_{\mathbf{1}}, y_{1}\right),\left(\mathbf{x}_{\mathbf{2}}, y_{2}\right), \cdots,\left(\mathbf{x}_{m}, y_{m}\right)$ consists of $m$ examples, where the domain of a class $y_{i}$ is described as $\{1,2, \cdots, c\}$, and $\mathbf{x}_{i}$ is a vector in an $n$-dimensional space. An example weight of $\left(\mathbf{x}_{\mathbf{i}}, y_{i}\right)$ is represented as $D_{t}(i, y)$, where $t$ is the number of rounds and $t=1,2, \cdots, T$. An initial value for an example weight $D_{1}(i, y)$ is given by

$$
D_{1}(i, y)=\frac{1}{m c}
$$

An example weight is updated based on the prediction $h_{t}(\mathbf{x}, y)$ of a weak hypothesis $h_{t}$, which is obtained by a weak learning algorithm, for the class $y$ of an instance $\mathbf{x}$. Here $h_{t}(\mathbf{x}, y)$ outputs 1 or -1 as a predicted class. In this paper, we employ a decision stump which represents a decision tree of depth one as a weak learner. In AdaBoost.M2, a pseudo-loss $\epsilon_{t}$ of a weak hypothesis $h_{t}$ is obtained for all examples $i=1,2, \cdots, m$ and all classes $y=1,2, \cdots, c$.

$$
\epsilon_{t}=\frac{1}{2} \sum_{i=1}^{m} \sum_{y=1}^{c}\left(1-h_{t}\left(\mathbf{x}_{i}, y_{i}\right)+h_{t}\left(\mathbf{x}_{i}, y\right)\right)
$$

From this, $\beta_{t}$ is obtained as follows.

$$
\beta_{t}=\frac{\epsilon_{t}}{1-\epsilon_{t}}
$$

The example weight is updated to $D_{t+1}(i, y)$ based on $\beta_{t}$, where $Z_{t}$ represents the add-sum of all example weights and is employed to normalize example weights.

$$
\begin{aligned}
& D_{t+1}(i, y)=\frac{D_{t}(i, y)}{Z_{t}} \beta_{t}^{\frac{1}{2}\left(1+h_{t}\left(\mathbf{x}_{i}, y_{i}\right)-h_{t}\left(\mathbf{x}_{i}, y\right)\right)} \\
& \text { where } Z_{t}=\sum_{i=1}^{m} D_{t}(i, y) \beta_{t}^{\frac{1}{2}\left(1+h_{t}\left(\mathbf{x}_{i}, y_{i}\right)-h_{t}\left(\mathbf{x}_{i}, y\right)\right)}
\end{aligned}
$$


AdaBoost.M2 iterates this procedure $T$ times to construct $T$ weak hypotheses. The final classification model, which is given by (6), predicts the class of each example by a weighted vote of $T$ weak hypotheses, where a weight of a weak hypothesis $h_{t}$ is given by $\log \left(1 / \beta_{t}\right)$.

$$
h_{\mathrm{fin}}(\mathbf{x})=\arg \max _{y} \sum_{t=1}^{T}\left(\log \frac{1}{\beta_{t}}\right) h_{t}(\mathbf{x}, y)
$$

Experimental results show that AdaBoost.M2 exhibits high accuracy. However, it is relatively time-consuming even if it employs a decision stump as a weak learner since its time complexity is given by $O\left(2^{c} T m n\right)$, where $n$ is the number of attributes.

\section{$3 \quad$ Fast Learning Based on Data Squashing}

\section{$3.1 \quad$ BIRCH}

The main stream of conventional data mining research has concerned how to scale up a learning/discovery algorithm to cope with a huge amount of data. Contrary to this approach, data squashing [3] concerns how to scale down such data so that they can be dealt by a conventional algorithm. Here we show a data squashing method used in BIRCH [11], which represents a fast clustering [5] algorithm. We will modify this data squashing method in the next section as a basis of our method.

Data reduction methods can be classified into feature selection [6] and instance selection [7]. In machine learning, feature selection has gained greater attention since it is more effective in improving time-efficiency. We, however, have adopted instance selection since crucial information for classification is more likely to be lost with feature selection than instance selection, and instance selection can deal with massive data which do not fit in memory.

BIRCH takes a training data set $\mathbf{x}_{\mathbf{1}}, \mathbf{x}_{\mathbf{2}}, \cdots, \mathbf{x}_{m}$ as input, and outputs its partition $\gamma_{1}, \gamma_{2}, \cdots, \gamma_{n+1}$, where each of $\gamma_{1}, \gamma_{2}, \cdots, \gamma_{n}$ represents a cluster, and $\gamma_{n+1}$ is a set of noise. A training data set is assumed to be so huge that it is stored on a hard disk, and cannot be dealt by a global clustering algorithm. Data squashing, which transforms a given data set to a much smaller data set by abstraction, can be considered to speed up learning in this situation. BIRCH squashes the training data set stored on a hard disk to obtain a CF (clustering feature) tree, and applies a global clustering algorithm to squashed examples each of which is represented by a leaf of the tree.

A CF tree represents a height-balanced tree which is similar to a $\mathrm{B}+$ tree [2]. A node of a $\mathrm{CF}$ tree represents a $\mathrm{CF}$ vector, which corresponds to an abstracted expression of a set of examples. For a set of examples $\mathbf{x}_{\mathbf{1}}, \mathbf{x}_{\mathbf{2}}, \cdots, \mathbf{x}_{N_{\phi}}$ to be squashed, a CF vector $\mathbf{C F}_{\phi}$ consists of the number $N_{\phi}$ of examples, the add-sum vector $\mathbf{L} \mathbf{S}_{\phi}$ of examples, and the squared-sum $S S_{\phi}$ of attribute values of examples. 


$$
\begin{aligned}
\mathbf{C F}_{\phi} & =\left(N_{\phi}, \mathbf{L} \mathbf{S}_{\phi}, S S_{\phi}\right) \\
\mathbf{L} \mathbf{S}_{\phi} & =\sum_{i=1}^{N_{\phi}} \mathbf{x}_{i} \\
S S_{\phi} & =\sum_{i=1}^{N_{\phi}}\left\|\mathbf{x}_{i}\right\|^{2}
\end{aligned}
$$

Since the CF vector satisfies additivity and can be thus updated incrementally, $\mathrm{BIRCH}$ requires only one scan of the training data set. Moreover, various intercluster distance measures can be calculated with the corresponding two $\mathrm{CF}$ vectors only. This signifies that the original data set need not be stored, and clustering can be performed with their CF vectors only.

A CF tree is constructed with a similar procedure for a $\mathrm{B}+$ tree. When a new example is read, it follows a path from the root node to a leaf, then nodes along this path are updated. Selection of an appropriate node in this procedure is based on a distance measure which is specified by a user. The example is assigned to its closest leaf if the distance between the new example and the examples of the leaf is below a given threshold $L$. Otherwise the new example becomes a novel leaf.

\subsection{Application of Data Squashing to Classification and Regression}

DuMouchel proposed to add moments of higher orders to the CF vector, and applied his data squashing method to regression [3]. Pavlov applied data squashing to support vector machine: a classifier which maximizes margins of training examples under a similar philosophy to boosting [9]. Nakayasu substituted a product-sum matrix for the $\mathrm{CF}$ vector, and applied their method to Bayesian classification [8]. They proposed a tree structure similar to the CF tree, and defined the squared add-sum of eigenvalues of the covariance matrix for each squashed example as information loss.

\section{Proposed Method}

\subsection{SB Loop}

Data squashing, which we explained in the last section, typically represents a single squashing of a training data set based on a distance measure. Several pieces of work including that of Nakayasu [8] consider how examples are distributed, and can be considered to squash a data set more appropriately than an approach based on a simple distance measure. However, we believe that a single squashing can consider distribution of examples only insufficiently.

In order to cope with this problem, we propose to squash the training data set iteratively. Since a boosting procedure outputs a set of example weights each 
of which represents difficulty of prediction of the corresponding example, we considered to use them in data squashing. By using these example weights, we can expect that examples which are difficult to be predicted would be squashed moderately, and examples which are easy to be predicted would be squashed excessively. Alternatively, our approach can be viewed as a speed-up of AdaBoost.M2 presented in section 2 with small degradation of accuracy.

Note that a simple application of a CF tree, which was originally proposed for clustering, would squash examples belonging to different classes to an identical squashed example. We believe that such examples should be processed separately, and thus propose an SF (Squashed-Feature) tree which separates examples belonging to different classes in its root node, and builds a CF tree from each child node. Figure 1 shows an example of an SF tree for a 3-class classification problem.

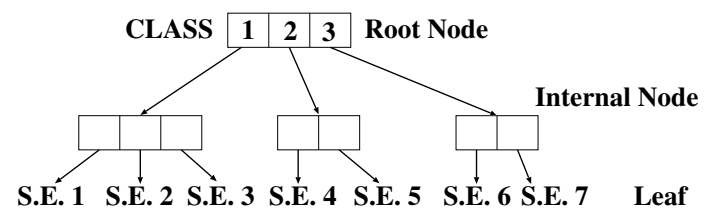

Fig. 1. An example of an Squashed-Feature tree, where an S.E. represents a squashed example

Our approach below iteratively squashes the training data set based on the set of example weights which are obtained from a boosting procedure. Since this squashing and boosting procedure is iterated so that the training data set is squashed appropriately, we call our approach an SB (Squashing-Boosting) loop.

1. Initial data squashing

Given $m$ examples $\left(\mathbf{x}_{\mathbf{1}}, y_{1}\right),\left(\mathbf{x}_{\mathbf{2}}, y_{2}\right), \cdots,\left(\mathbf{x}_{m}, y_{m}\right)$, obtain $p$ squashed examples $\left(\mathbf{x}_{\text {sub } 1}, y_{\text {sub } 1}\right),\left(\mathbf{x}_{\text {sub } 2}, y_{\text {sub } 2}\right), \cdots,\left(\mathbf{x}_{\text {sub } p}, y_{\text {sub } p}\right)$ by constructing an SF tree. The threshold $L$ for judging whether an example belongs to a leaf is uniformly settled to $L_{0}{ }^{1}$.

2. For $\theta=1$ until $\theta=\Theta$ step 1

(a) Application of boosting

Apply AdaBoost.M2 to $\left(\mathbf{x}_{\text {sub } 1}, y_{\text {sub } 1}\right),\left(\mathbf{x}_{\text {sub } 2}, y_{\text {sub } 2}\right), \cdots,\left(\mathbf{x}_{\text {sub } p}\right.$, $\left.y_{\text {sub }}\right)$, and obtain example weights $D_{T}\left(1, y_{\text {sub } 1}\right), D_{T}\left(2, y_{\text {sub } 2}\right), \cdots$, $D_{T}\left(p, y_{\text {sub }} p\right)$ of the final round $T$ and a classification model.

(b) Update of thresholds

For a leaf which represents a set of examples $\left(\mathbf{x}_{\text {sub } i}, y_{\text {sub } i}\right)$, update its

\footnotetext{
${ }^{1}$ As we explained in section 3.1, BIRCH employs a threshold $L$ to judge whether an example belongs to a leaf, i.e. a squashed example.
} 
threshold $L\left(\theta, \mathbf{x}_{\text {sub } i}\right)$ to $L\left(\theta+1, \mathbf{x}_{\text {sub } i}\right)$.

$$
L\left(\theta+1, \mathbf{x}_{\text {sub i }}\right)=L\left(\theta, \mathbf{x}_{\text {sub i }}\right) \frac{D_{1}(i, y)}{D_{T}(i, y)} \log a(\theta, i)
$$

where $D_{1}(i, y)$ is given by $(1)$, and $a(\theta, i)$ represents the number of examples which are squashed into the leaf $i$.

(c) Data Squashing

Construct a novel SF tree from the training examples. In the construction, if a leaf has a corresponding leaf in the previous SF tree, use $L\left(\theta+1, \mathbf{x}_{\text {sub i }}\right)$ as its threshold. Otherwise, use $L_{0}$ as its threshold.

3. Output the current classification model.

In each iteration, a squashed example with a large example weight is typically divided since we employ a smaller threshold for the corresponding leaf node. On the other hand, a squashed example with a small example weight is typically merged with other squashed examples since we employ a larger threshold for the corresponding leaf node. We show a summary of our SB loop in figure 2.

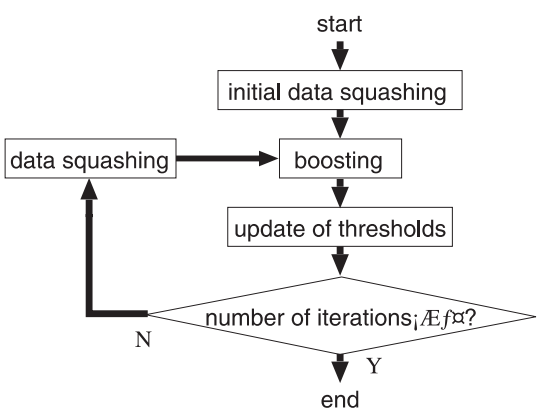

Fig. 2. SB (Squashing-Boosting) loop

\subsection{Projected SVD Distance}

BIRCH employs a distance measure such as average cluster distance and Euclidean distance in constructing a CF tree [11]. These distance measures typically fail to represent distribution of examples since they neglect interactions among attributes.

In order to circumvent this problem, we propose to store the number of examples $N_{\phi}$, an average vector $\boldsymbol{\mu}_{\phi}$, and a quasi-product-sum matrix $W_{\phi}$ in a node $\phi$ of our SF tree, where $\boldsymbol{\mu}_{\phi}=\sum_{i=1}^{N_{\phi}} \mathbf{x}_{i} / N_{\phi}$. A quasi-product-sum matrix, which is given by (11), is updated when a novel example is squashed into its corresponding leaf. The update is done by adding the product-sum matrix of the novel example to the quasi-product-sum matrix. A quasi-product-sum matrix of 
an internal node is given by the add-sum of the quasi-product-sum matrices of its child nodes.

$$
W_{\phi}=\left(\begin{array}{ccccc}
g_{11 \phi} & \cdots & g_{1 j \phi} & \cdots & g_{1 m \phi} \\
\vdots & \ddots & & & \vdots \\
g_{i 1 \phi} & & g_{i j \phi} & & g_{i m \phi} \\
\vdots & & & \ddots & \vdots \\
g_{m 1 \phi} & \cdots & g_{m j \phi} & \cdots & g_{m m \phi}
\end{array}\right)
$$

$$
g_{i j \phi}=\left\{\begin{array}{l}
\sum_{k} g_{i j k} \\
\text { for an internal node, where } k \text { represents an identifier of its child } \\
\text { nodes } \\
x_{f i} x_{f j}+g_{i j \phi}^{\prime} \\
\text { for a novel example, where } x_{f i} \text { represents an attribute value of an } \\
\text { attribute } i \text { for an inputted example } f, \text { and } g_{i j \phi}^{\prime} \text { represents the } \\
\text { original value of a squashed example } \phi)
\end{array}\right.
$$

Our projected SVD distance $\Delta\left(\mathbf{x}_{i}, k\right)$ between an example $\mathbf{x}_{i}$ and a squashed example $k$ is defined as follows.

$$
\Delta\left(\mathbf{x}_{i}, k\right)=\left(\mathbf{x}_{i}-\boldsymbol{\mu}_{k}\right)^{t} S_{k}^{-1}\left(\mathbf{x}_{i}-\boldsymbol{\mu}_{k}\right)
$$

where $S_{k}$ represents the quasi-covariance matrix obtained from $W_{k}$.

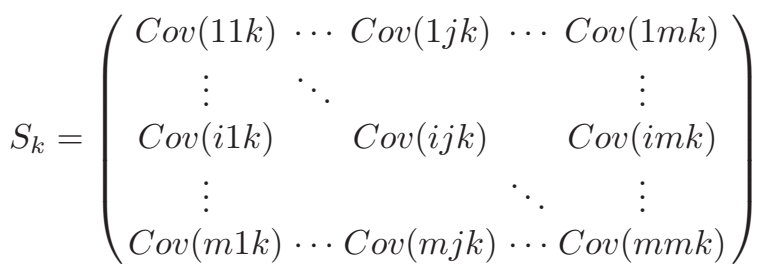

$$
\begin{aligned}
& \text { where } \operatorname{Cov}(i j k)=\frac{g_{i j k}}{N_{k}}-E(i k) E(j k) \\
& \text { and } E(i k) \text { is the } i \text { th element of } \boldsymbol{\mu}_{k}
\end{aligned}
$$

Our projected SVD distance requires the inverse matrix of $S$, and we use singular value decomposition [10] for this problem. In the method, $S$ is represented as a product of three matrices as follows.

$$
S=U \cdot\left(\begin{array}{llll}
z_{1} & & & 0 \\
& z_{2} & & \\
& & \cdots & \\
& & & z_{n}
\end{array}\right) \cdot V
$$

where $U$ and $V$ are orthogonal matrices. Consider two vectors $\mathbf{x}$, b which satisfy $S \cdot \mathbf{x}=\mathbf{b}$. If $S$ is singular, there exists a vector $\mathbf{x}^{\prime}$ which satisfies $S \cdot \mathbf{x}^{\prime}=0$. 
In general, there are an infinite number of $\mathbf{x}$ which satisfies $S \cdot \mathbf{x}=\mathbf{b}$, and we choose the one with minimum $\left\|x^{2}\right\|$ as a representative. For this we use

$$
\mathbf{x}=V \cdot\left[\operatorname{diag}\left(1 / z_{j}\right)\right] U^{T} \cdot \mathbf{b},
$$

where we settle $1 / z_{j}=0$ if $z_{j}=0$, and $\operatorname{diag}\left(1 / z_{j}\right)$ represents a diagonal matrix of which $j$ th element is $1 / z_{j}$. This is equivalent to obtaining $\mathbf{x}$ which minimizes $\|S \cdot \mathbf{x}-\mathbf{b}\|$, i.e. an approximate solution for $S \cdot \mathbf{x}=0[10]$.

\section{$5 \quad$ Experimental Evaluation}

\subsection{Experimental Condition}

We employ artificial data sets as well as real-world data sets in the experiments. Each of our artificial data sets contains, as classes, four normal distributions with equal variances and the covariances are 0 . We show means and variances of the classes in table 1 . We varied the number of attributes 3, 5, 10. Each class contains 5000 examples. In the experiments for evaluating our SF tree, the number of examples for each class was settled to 500 in order to investigate on the cases with a small number of examples.

Table 1. Means and variances of classes in the artificial data sets, where $\mu_{i}$ represents the mean of an attribute $i$ for each class

\begin{tabular}{|c||c|c|c|c|c|c|c|c|c|c|c|}
\hline class & $\mu_{1}$ & $\mu_{2}$ & $\mu_{3}$ & $\mu_{4}$ & $\mu_{5}$ & $\mu_{6}$ & $\mu_{7}$ & $\mu_{8}$ & $\mu_{9}$ & $\mu_{10}$ & variance \\
\hline \hline 1 & -6 & 2 & -9 & 3 & 10 & 5 & -4 & -10 & 2 & 9 & 7 \\
\hline 2 & 7 & -2 & 0 & 10 & 3 & -4 & 4 & -7 & 3 & 7 & 9 \\
\hline 3 & -2 & -3 & -9 & -6 & 3 & 8 & 8 & 1 & -2 & -3 & 5 \\
\hline 4 & -5 & -5 & 8 & 5 & 1 & -7 & 6 & 6 & 7 & -6 & 8 \\
\hline
\end{tabular}

We employed the KDD Cup 1999 data set [1], from which we produced several data sets. Since it is difficult to introduce a distance measure of data squashing for a nominal attribute and binary attributes can be misleading in calculating a distance, we deleted such attributes before the experiments. As the result, each data set contains 12 attributes instead of 43 . We selected the normal-access class and the two most frequent fraudulent-access classes, and defined a 3-class classification problem. We have generated ten data sets by choosing 10000, 20000, *. 90000, and 97278 examples from each class.

We measured classification accuracy and computational time using 5-fold cross-validation. For artificial data sets, we have chosen boosting without data squashing and boosting with a single data squashing in order to investigate on effectiveness of our approach. We also evaluated our projected SVD distance by 
comparing it with average cluster distance and Euclidean distance. The threshold $L$ was settled so that the number of squashed examples becomes approximately $3 \%$ of the number of examples, the number of iterations in boosting was settled to $T=100$, and the number of iterations of data squashing in our approach was $\Theta=3$. For real-world data sets, we compared our projected SVD distance with average cluster distance. We omitted Euclidean distance due to its poor performance in the artificial data sets.

In the experiments for evaluating tree structures in data squashing, we employed our SF tree and a tree which squashes examples without class information. The experiments were performed with average cluster distance and Euclidean distance since we considered that the small number of examples favors simple distance measures. Since the latter tree can squash examples of different classes into an example, the class of a squashed example was determined by a majority vote. In these experiments, we settled $L$ so that the number of squashed examples becomes approximately $10 \%$ of the number of examples, and we used $T=100, \Theta=5$.

\subsection{Experimental Results and Analysis}

Artificial Data Sets We show the results with our projected SVD distance in figure 3. From the figure, we see that our SB loop, compared with boosting with single data squashing, exhibits higher accuracy (approximately $8 \%$ ) though its computational time is 5 to 7 times longer for almost all data sets. These results show that a single data squashing fails to squash data appropriately, while our SB loop succeeds in doing so by iteratively refining the squashed data sets. Moreover, degradation of accuracy for our approach, compared with boosting without data squashing is within $3 \%$ except for the case of 10 attributes, and our method is 5 to 6 times faster. These results show that our data squashing is effective in speeding-up boosting with a little sacrifice in accuracy.

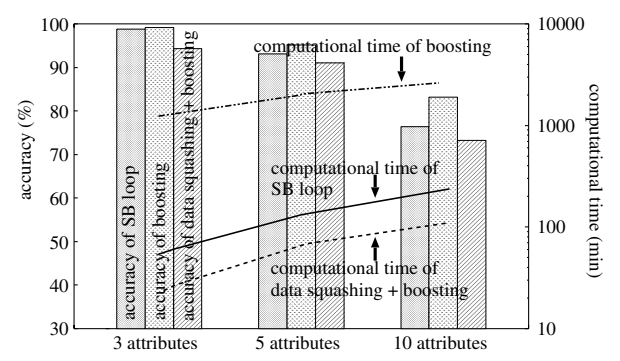

Fig. 3. Effect of SB loop with projected SVD distance for the artificial data sets

We also show the results with average cluster distance and Euclidean distance in figure 4 . The figure shows that our approach is subject to large degradation of accuracy compared with boosting, especially when Euclidean distance 
is employed. These results justify our projected SVD distance, which reflects distribution of examples to distance.

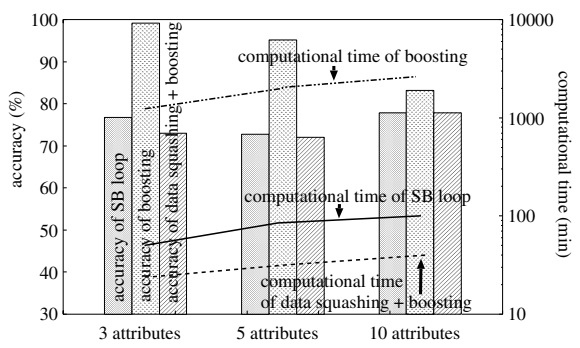

(a)

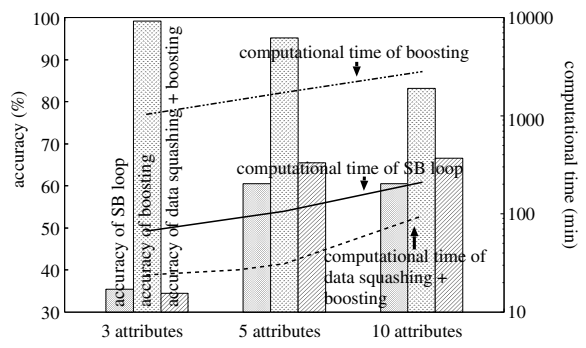

(b)

Fig. 4. Effect of SB loop for the artificial data sets with average inter-cluster distance (a) and Euclidean distance (b)

Real-World Data Sets We show experimental results with our projected SVD distance and average cluster distance in figure 5 . In both cases, compared with boosting with single data squashing, SB loop exhibits approximately $8 \%$ of improvement in accuracy in average though its computational time is approximately 4 to 6 times longer. Compared with boosting without data squashing, when our projected SVD distance is employed, our approach shortens computational time at most to $1 / 35$ with a small degradation in accuracy. Moreover, the accuracy of our SB loop is no smaller than $92 \%$ when our projected SVD distance is employed. The good performance of our approach can be explained from characteristics of the data set. In the data set, two attributes have large variances which are more than 10000 times greater than the variances of the other attributes. Therefore, data squashing is practically performed in terms of these attributes, and is relatively easier than the cases with the artificial data sets. Moreover, these attributes are crucial in classification since our approach sometimes improves accuracy of boosting without data squashing.

Effectiveness of an SF Tree We show results with the artificial data sets in terms of tree structures and distance measures in figure 6. Regardless of distance measures, our SF tree ((a) and (c)) typically exhibits high accuracy with our SB loop. We attribute the reason to appropriate data squashing. On the contrary, neglecting class information in data squashing ((b) and (d)) typically lowers accuracy, especially when we use data squashing iteratively. We consider that these results justify our SF tree.

In terms of average computational time, our SF tree is approximately five times longer than the tree which neglects class information with average cluster 


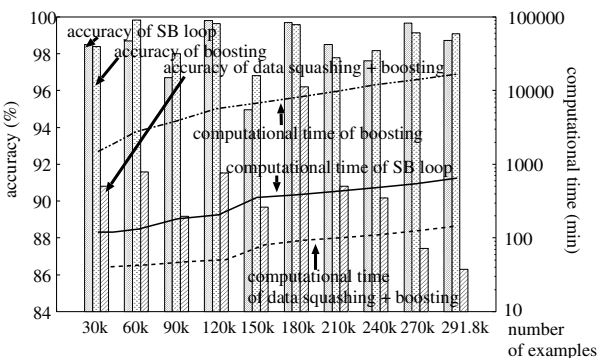

(a)

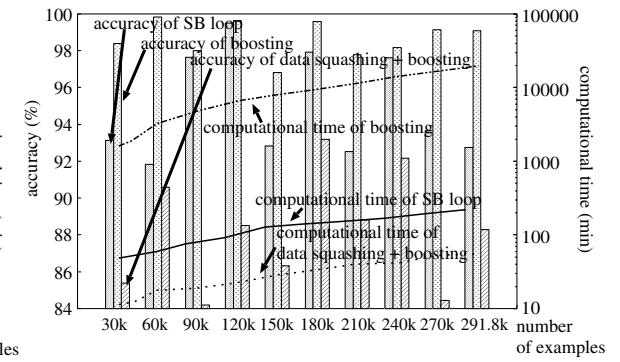

(b)

Fig. 5. Results of the KDD Cup 1999 data with projected SVD distance (a) and average cluster distance (b)

distance, and is almost equivalent to the tree which neglects class information with Euclidean distance. This can be explained by the number of squashed examples since our SF tree has, in average, approximately 4.95 and 1.15 times of squashed examples than the tree which neglects class information.

\section{Conclusion}

The main stream of conventional data mining research has concerned how to scale up a learning/discovery algorithm to cope with a huge amount of data. Contrary to this approach, data squashing [3] concerns how to scale down such data so that they can be dealt by a conventional algorithm.

Our objective in this paper represents a speed-up of boosting based on data squashing. In realizing the objective, we have proposed a novel method which iteratively squashes a given data set using example weights obtained in boosting. Moreover, we have proposed, in data squashing, the projected SVD distance measure, which tries to reflect distribution of examples to distance. Lastly, our $\mathrm{SF}$ tree considers class information in data squashing unlike the CF tree used in BIRCH [11].

We experimentally compared our approach with boosting without data squashing and boosting with a single data squashing using both artificial and real-world data sets. Results show that our approach speeds-up boosting 5 to 6 times while its degradation of accuracy was typically less than approximately 3 $\%$ for artificial data sets. Compared with boosting with a single data squashing, our approach requires 5 to 7 times of computational time, but improves accuracy approximately $8 \%$ in average. For the real-world data sets from the KDD Cup 1999 data set, our projected SVD distance exhibits approximately $8 \%$ of higher accuracy in average compared with average cluster distance, while the required computational time is almost the same. Considering class information in our SF tree improves accuracy approximately $2.4 \%$ to $27 \%$ in average when the number of examples is small. 


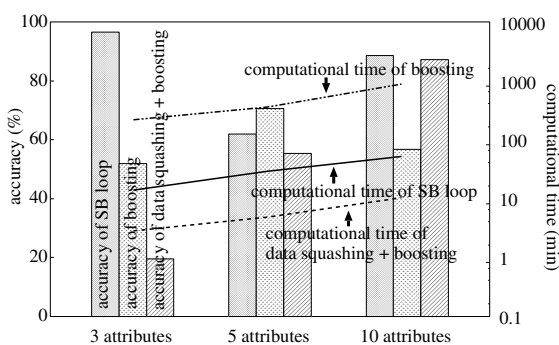

(a)

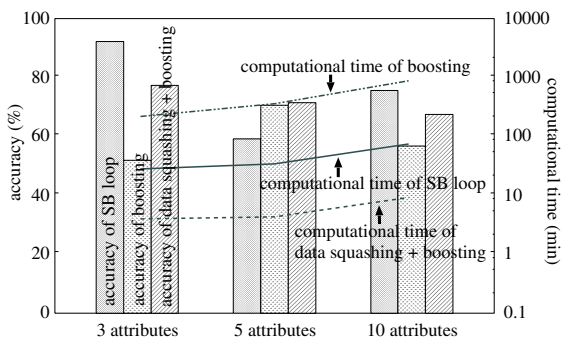

(c)

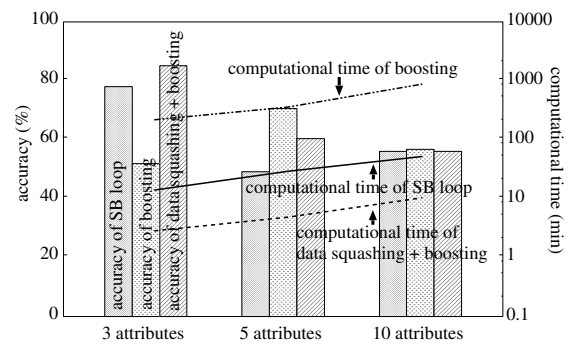

(b)

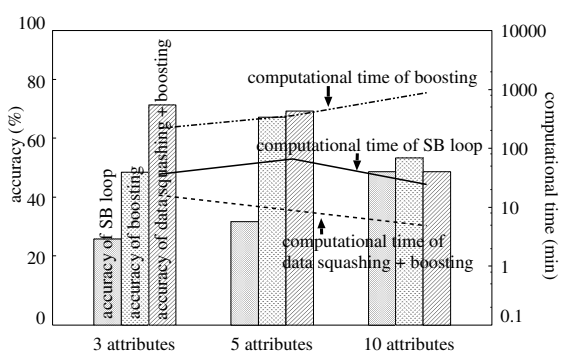

(d)

Fig. 6. Effectiveness of an SF tree with average cluster distance (a), a tree which ignores class information with average cluster distance (b), an SF tree with Euclidean distance (c), and a tree which ignores class information with Euclidean distance $(d)$

\section{References}

1. Bay, S.: UCI KDD Archive, http://kdd.ics.uci.edu/, Dept. of Information and Computer Sci., Univ. of California Irvine. (1999). 93

2. Comer, D.: The Ubiquitous B-Tree, ACM Computing Surveys, Vol. 11, No. 2, pp. $121-137$ (1979). 88

3. DuMouchel, W. et al.: Squashing Flat Files Flatter, Proc. Fifth ACM Int'l Conf. on Knowledge Discovery and Data Mining (KDD), pp. 6-15 (1999). 86, 88, 89, 96

4. Freund, Y. and Schapire, R. E.: Experiments with a New Boosting Algorithm, Proc. Thirteenth Int'l Conf. on Machine Learning (ICML), pp. 148-156 (1996). 86

5. Kaufman, L. and Rousseeuw, P. J.: Finding Groups in Data, Wiley, New York (1990). 88

6. Liu, H. and Motoda, H.: Feature Selection, Kluwer, Norwell, Mass. (1998). 88

7. Liu, H. and Motoda, H. (eds.): Instance Selection and Construction for Data Mining, Kluwer, Norwell, Mass. (2001). 88

8. Nakayasu, T., Suematsu, N., and Hayashi, A.: Learning Classification Rules from Large-Scale Databases, Proc. 62th Nat'l Conf. of Information Processing Society of Japan, Vol. 2, pp. 23-24 (2001, in Japanese). 89

9. Pavlov, D., Chudova, D., and Smyth, P.: Towards Scalable Support Vector Machines Using Squashing, Proc. Sixth ACM Int'l Conf. on Knowledge Discovery and Data Mining (KDD), pp. 295-299 (2000). 89 
10. Press, W. H. et al.: Numerical Recipes in $C$ - Second Edition, Cambridge Univ. Press, Cambridge, U. K. (1992). 92, 93

11. Zhang, T., Ramakrishnan, R., and Livny, M.: BIRCH: An Efficient Data Clustering Method for Very Large Databases, Proc. 1996 ACM SIGMOD Int'l Conf. on Management of Data (SIGMOD), pp. 103-114 (1996). 88, 91, 96 